\title{
A $\Delta \Sigma$ Modulator Automated Synthesis Tool for Wireless Standards
}

\author{
Houda DAOUD, Samir Ben SALEM, Sawssan LAHIANI, Mourad LOULOU
}

\begin{abstract}
This paper presents the prediction of single-bit discrete-time feed-forward Delta-Sigma (DT FF $\Delta \Sigma$ ) modulators performance for wireless standards with the use of two methods. The presented work uses the MAPLE tool and a new design automation tool to estimate the performance of different DT FF $\Delta \Sigma$ modulators topologies intended for low power consumption systems. The proposed tool is based on synthesis algorithm, which takes advantage of analytical models of both FF $\Delta \Sigma$ modulator and operational transconductance amplifier (OTA) performance. By defining the required specifications, the proposed synthesis tool is capable to find the predictive performance of both the modulator topology and the required OTA building block for future process. A Graphical User Interface is programmed to easily present some designed circuits examples.
\end{abstract}

Keywords: DT FF $\Delta \Sigma$ modulators performance prediction; $\Delta \Sigma$ modulator and OTA performance modelling; design automation; programmed Graphical User Interface; wireless standards

\section{INTRODUCTION}

Among the diversity of Delta-Sigma $(\Delta \Sigma)$ modulators architectures, feed-forward Delta-Sigma (FF $\Delta \Sigma$ ) modulators have become the most used architectures to meet the requirements of wireless communication systems [1]. In fact, it has recently become very popular to feedforward the input signal in wideband $\Delta \Sigma$ modulators, so that the integrators only process quantization errors, the advantage being that the actual signal is not distorted by operational amplifier and integrator nonlinearities [2]. In addition, the benefits of these topologies are their abilities to achieve high resolutions with a decrease in power consumption [3]. The trend of designing low power $\Delta \Sigma$ modulators using upcoming CMOS Nano-process has been growing rapidly in recent years [4]. Designers usually require much time to select the best $\Delta \Sigma$ modulator architecture with the appropriate process node, to determine its performance and to acquire the desired operational transconductance amplifier (OTA) circuits. The time spent for designing is very long and needs a lot of experience. Thus, new tools for $\Delta \Sigma$ modulators are required for unskilled designers.

In front of the increasing complexity of $\Delta \Sigma$ modulators, several studies proposed many tools $[5,6]$. Medeiro proposed a high-level specifications tool. His tool has used an equation-based approach at the modulator level and a simulation-based approach at the cell level. It has only supported single-loop $\Delta \Sigma$ modulators and the simulation has taken a lot of time [7]. Tang and Doboli presented a topology-synthesis methodology which gives all possible topologies under various design considerations for single-loop single-bit $\Delta \Sigma$ modulators [8]. However, the $\Delta \Sigma$ modulator topology performance is strongly affected by the non-idealities of building blocks, therefore, the implementation is even more difficult in nanometer CMOS technologies. For this reason, it is recommended to ameliorate or to present other $\Delta \Sigma$ modulators structures with high performances that satisfy the specifications of new standards.

Given the frequent use of submicron CMOS process for low power $\Delta \Sigma$ modulators design, the aim of this work is focused on the prediction of different structures of DT
FF $\Delta \Sigma$ modulators performance with process scaling for various mobile telecommunications standards.

In this context, this paper proposes a novel helpful approach for the synthesis of low power FF $\Delta \Sigma$ modulators with Nanometric CMOS process. Some equations models linking the $\mathrm{FF} \Delta \Sigma$ modulator performance to the design and the technological parameters are developed. Then, a Graphical User Interface (GUI) is programmed in which the user can get access to additional interfaces for different tasks. The next Section details the procedure of NanoCMOS $\Delta \Sigma$ modulators performance prediction including the modeling of $\Delta \Sigma$ modulator and OTA performance and the use of the MAPLE tool. The following Section presents the design automation approach and the proposed synthesis algorithm. Some design examples are provided afterwards in order to show the operation of the system. Finally, the conclusions drawn from this work and possible future works are presented.

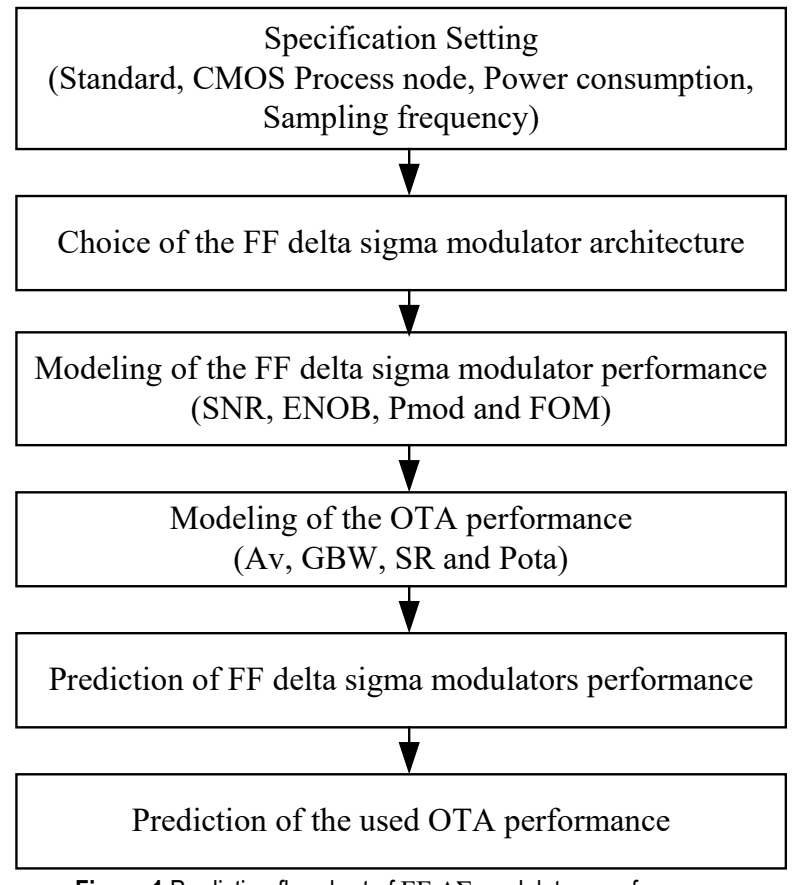

Figure 1 Prediction flowchart of FF $\Delta \Sigma$ modulators performance 


\section{PROCEDURE OF NANOCMOS FF $\triangle \Sigma$ MODULATORS PERFORMANCE PREDICTION}

In this Section, a prediction flowchart is introduced to detail the different steps to acquire predictive FF $\Delta \Sigma$ modulators performance. As it can be seen from Fig. 1, firstly, we fix the specifications including the wireless standard, the CMOS process node, the power consumption and the $F_{\mathrm{S}}$. Secondly, by varying the order of the FF $\Delta \Sigma$ modulator, we opt for its architecture. Taking both design and technological parameters into consideration, some equations models related to FF $\Delta \Sigma$ modulator and OTA performance could be developed. Finally, the performance of FF $\Delta \Sigma$ modulators architectures and the required OTA circuit performance are predicted for future process.

\subsection{Modelling of FF $\Delta \Sigma$ Modulator Performance}

Currently, designers focus on DT FF $\Delta \Sigma$ modulators design for submicron process to meet the requirements of new wireless communications systems [9]. Our objective consists of predicting the performance of several singlebits DT FF $\Delta \Sigma$ modulators structures namely the signal-tonoise ratio $(S N R)$, the resolution $(E N O B)$, the power consumption $\left(P_{\bmod }\right)$ and the figure of merit $(F O M)$ for wireless standards. To estimate this performance, the selected FF $\Delta \Sigma$ modulator architecture should be modelled accurately. When modelling, we suppose that the errors caused by non idealities affecting the analog building blocks are neglected.

\subsubsection{Signal to Noise Ratio $(S N R)$}

The SNR is the most used criterion to characterize the modulator performance. In fact, the $S N R$ which represents the ratio between the input signal power $\left(P_{\mathrm{S}}\right)$ and the quantization noise power $\left(P_{\mathrm{Q}}\right)$ is defined by the following equation:

$S N R=10 \cdot \log \left(\frac{P_{\mathrm{S}}}{P_{\mathrm{Q}}}\right)=10 \cdot \log \left(\frac{U_{\max }^{2}}{2 P_{\mathrm{Q}}}\right)$,

$U_{\max }$ presents the maximum voltage of the signal applied to the modulator input. It is given by Eq. (2):

$U_{\max }=V_{\text {ref }} \cdot O L$,

$O L$ and $V_{\text {ref }}$ represent respectively the overload and the reference voltage. The quantization noise power is given by the following relationship [10]:

$P_{\mathrm{Q}}=\int_{-f_{\mathrm{b}}}^{+f_{\mathrm{b}}} \frac{\Delta^{2}}{12 F_{\mathrm{S}}}|N T F(f)|^{2} \mathrm{~d} f$,

where $\Delta, F_{\mathrm{S}}$ and $N T F$ are respectively the quantization step, the $F_{\mathrm{S}}$ and the noise transfer function. $P_{\mathrm{Q}}$ varies from one modulator architecture to another. If we use a $L^{\text {th }}$-order $\Delta \Sigma$ modulator architecture where its NTF is in the form of $(1-$ $\left.z^{-1}\right)^{L}$, Eq. (3) is approximately rewritten as follows:

$$
P_{\mathrm{Q}} \approx \frac{\Delta^{2}}{12}\left(\frac{\pi^{2 L}}{(2 L+1) O S R^{(2 L+1)}}\right)
$$

where $L$ and $O S R$ are respectively the order and the over sampling ratio. The quantization step $\Delta$ is given by:

$\Delta=\frac{V_{\mathrm{fs}}}{2^{B}-1}$

where $V_{\mathrm{fs}}$ and $B$ are the full scale voltage and the quantizer bits number. For a $N$-stage cascaded $\Delta \Sigma$ modulator architecture, the quantization noise power can be approximated to Eq. (6):

$$
P_{\mathrm{Q}} \approx d_{2 N-3}^{2}\left(\frac{\Delta_{N}^{2}}{12}\right)\left(\frac{\pi^{2 L}}{(2 L+1) O S R^{(2 L+1)}}\right),
$$

with $d_{2 N-3}$ being the digital coefficient of the $N^{\text {th }}$-stage $\Delta \Sigma$ modulator.

\subsubsection{Effective Number of Bits $(E N O B)$}

The quantization levels number of a conversion system is defined by the effective number of bits $(E N O B)$ or resolution. The resolution is expressed as [11]:

$$
E N O B=\frac{S N R(\mathrm{~dB})-1,76}{6,02}
$$

\subsubsection{Power Consumption}

Given the increasing number of both the portable equipment and the functional blocks integrated on the same chip, low power consumption of a $\Delta \Sigma$ modulator is an important property. In this work, we aim to design a modulator structure where the power consumption of the used comparator is very low. In [12], Feldman assumes that the power dissipated in a switched capacitor integrator is that which is required to charge and discharge the sampling capacitor $C_{\mathrm{S}}$. Then, the required power consumption of the modulator can be approximated to Eq. (8):

$$
P_{\text {mod }}>>\sum P_{\text {integ }}>>\sum\left(V_{\mathrm{dd}}^{2} \cdot C_{\mathrm{S}} \cdot F_{\mathrm{S}}\right)
$$

$V_{\text {dd }}, C_{\mathrm{S}}$ and $F_{\mathrm{S}}$ are respectively the supply voltage, the sampling capacitor and the $F_{\mathrm{S}}$.

\subsubsection{Signal bandwidth (BS)}

It is the frequency band on which the $\Delta \Sigma$ modulator may be used. It can be written in the following form [11]:

$$
B S=\frac{F_{\mathrm{S}}}{2 \cdot O S R}
$$

\subsubsection{Figure of Merit (FOM)}

The FOM compares the performance of various $\Delta \Sigma$ modulators. It is given by the following expression [13]: 
$F O M=\frac{P_{\text {mod }}}{2^{E N O B} \cdot 2 B S}$,

where $B S$ is the signal bandwidth.

\subsection{Modelling of OTA Performance}

The most important block in the DT FF $\Delta \Sigma$ modulator structure is the OTA circuit which has a great impact on modulators performance. Thus, the designer should use a suitable OTA topology to realize robust $\Delta \Sigma$ modulators. In this work, we focus on finding a relationship between $\Delta \Sigma$ modulator and OTA performance. The main OTA performance which may directly affect the operation of $\Delta \Sigma$ modulator are the DC gain $\left(A_{\mathrm{dc}}\right)$, the gain-bandwidth product $(G B W)$ and the Slew Rate $(S R)$.

\subsubsection{Gain Requirement}

The settling error at the output of the OTA circuit, resulting from the finite open loop DC gain $A_{\mathrm{dc}}$ is approximately given by [14-15]:

$e_{0}=\frac{1}{1+\left(\beta_{i} \cdot A_{\mathrm{dc}}\right)} \approx \frac{1}{\beta_{i} \cdot A_{\mathrm{dc}}}$,

where $\beta_{i}$ is the feedback factor.

The OTA circuit will be used in a data converter with $E N O B$ bits accuracy and it must amplify signals to within half least significant bits (LSB) of the ideal value [14]. Therefore, the required OTA DC gain can be obtained to meet the accuracy requirement of the ADC circuit. It is given by the following expression:

$A_{\mathrm{dc}} \geq \frac{2^{(E N O B+1)}}{\beta_{i}}$.

The minimum required DC open loop gain can be expressed as:

$$
A_{\mathrm{dc}}=\frac{2^{(E N O B+1)}}{\beta_{i}} .
$$

\subsubsection{Gain-Bandwidth Product Requirement}

For a single pole system, where the OTA settling requirement is $E N O B$ bits, the output settles to $E N O B$ in the sampling time period $T_{\mathrm{S}}$ if [16]:

$\mathrm{e}^{-\frac{T_{S}}{\tau}} \prec 2^{-E N O B}$

where $\tau$ and $T_{\mathrm{S}}$ are respectively the settling time constant and the $T_{\mathrm{S}}$. Eq. (15) describes the settling time constant where $C_{\mathrm{f}}$ is the integration capacitor and $\beta_{i}$ is the feedback factor which is detailed in Eq. (16) [17].

$\tau=\frac{1}{\beta_{i} \cdot G B W}$
$\beta_{i}=\frac{C_{\mathrm{f}}}{C_{\mathrm{S}}+C_{\mathrm{f}}}$

It leads to the following requirement for the $G B W$ using Eq. (14) and Eq. (15):

$G B W \geq E N O B\left(\frac{\ln (2)}{\beta_{i} \cdot T_{S}}\right)$

The minimum required OTA $G B W$ is given by Eq. (18):

$G B W_{\min }=F_{S} \cdot E N O B\left(\frac{\ln (2)}{\beta_{i}}\right)$

\subsubsection{Slew Rate Requirement}

It is assumed that the OTA integrator is a first order system, the maximum variation of the circuit response is given by the following expression [18]:

$\left.\frac{\mathrm{d}}{\mathrm{d} t}\left(V_{0}(t)\right)\right|_{t=0}=g_{1}\left(\frac{V_{\mathrm{in}, \max }}{\tau}\right)$ where $g_{1}=\frac{C_{\mathrm{S}}}{C_{\mathrm{f}}}$,

$g_{1}$ and $V_{\text {in, max }}$ are respectively the integrator gain and the maximum input voltage. Regarding the settling error, in the case of:

$S R \geq \frac{C_{\mathrm{S}}}{C_{\mathrm{f}}} \cdot \frac{V_{\mathrm{in}, \max }}{\tau}$

there is no Slew Rate limitation and the integrator output will be linearly settled. From Eq. (20), the minimum required OTA $S R$ is obtained:

$S R_{\min }=\frac{C_{\mathrm{S}}}{C_{\mathrm{f}}} \cdot \frac{V_{\mathrm{in}, \max }}{\tau}$.

Using Eq. (14), the minimum required Slew Rate is rewritten as:

$S R_{\text {min }}=F_{\mathrm{S}} \cdot E N O B \cdot \frac{C_{\mathrm{S}}}{C_{\mathrm{f}}} \cdot V_{\mathrm{in}, \max } \cdot \ln (2)$,

where $V_{\text {in, } \max }=V_{\text {ref }} \cdot O L$. The $V_{\text {ref }}$ voltage value is chosen very close to the OTA circuit output swing in order to increase the $\Sigma \Delta$ modulator input swing.

Referring to Eq. (13), Eq. (18) and Eq. (22), it can be noted that the OTA performance depends on the $\Delta \Sigma$ modulator performance (ENOB, OL, analog coefficient), the selected $F_{\mathrm{S}}$ and the CMOS process node $\left(V_{\mathrm{dd}}\right)$. The prediction of the required OTA circuit performance for each provided $\Delta \Sigma$ modulator architecture can be an important key to success. 


\subsection{Prediction of FF $\Delta \Sigma$ Modulator Performance through Maple Tool}

The presented work aims to estimate different FF $\Delta \Sigma$ modulators structures used for low and high-speed applications. The prediction is limited to single-bit FF $\Delta \Sigma$ modulator topology whose quantizer is a single-bit one $(B$ $=1)$. Therefore, according to the literature, we fix some standards summarized in Tab. 1 [19-21]. During this phase, to project some FF $\Delta \Sigma$ modulators performance predictions for low power systems, we choose a decreasing power consumption estimated less than $1 \mathrm{~mW}$ and a variable $F_{\mathrm{S}}$ using future CMOS process (Tab. 2). Tab. 2 presents supply voltages predicted for each process node using the Bisquare Weights method described in [22].

Table $1 \mathrm{ADC}$ specifications for mobile telecommunications standards

\begin{tabular}{|c|c|c|}
\hline Standards & Signal bandwidth (MHz) & Resolution (Bits) \\
\hline GSM & 0.1 & 13 \\
\hline Bluetooth & 1 & $10-12$ \\
\hline UMTS & 2 & 10 \\
\hline WCDMA & 3.84 & $9-12$ \\
\hline DVB-H & 7.6 & $9-10$ \\
\hline WLAN & 10 & $8-10$ \\
\hline WIMAX & 20 & $7-10$ \\
\hline
\end{tabular}

Table 2 Specifications

\begin{tabular}{|c|c|c|}
\hline $\begin{array}{c}\text { Process nodes }(\mathrm{nm}) \\
\text { /Supply voltage }(\mathrm{V})\end{array}$ & $\begin{array}{c}\text { Power consumption } \\
(\mathrm{mW})\end{array}$ & $\begin{array}{c}\text { Sampling frequency } \\
(\mathrm{MHz})\end{array}$ \\
\hline $22 / 0.68$ & 0.1 & \multirow{3}{*}{$20 \leq F_{\mathrm{S}} \leq 520$} \\
\hline $32 / 0.76$ & 0.2 & \\
\hline $45 / 0.86$ & 0.3 & \\
\hline $65 / 1.2$ & 0.4 & \\
\hline $90 / 1.2$ & 0.5 & \\
\hline $130 / 1.4$ & 0.6 & \\
\hline $180 / 1.8$ & 0.7 & \\
\hline $250 / 2.5$ & 0.8 & \\
\hline $350 / 3.3$ & 0.9 &
\end{tabular}

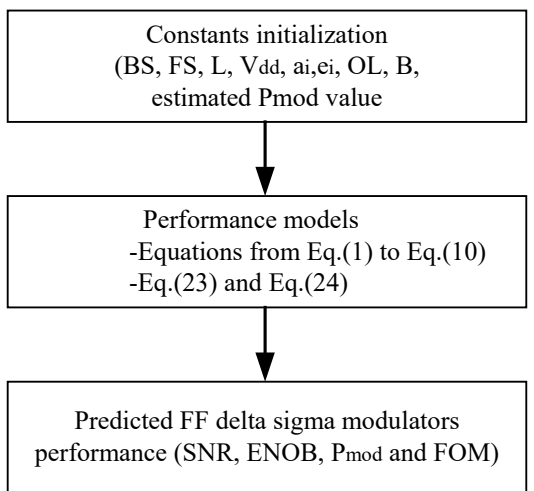

Figure 2 Prediction method of FF $\Delta \Sigma$ modulators performance through maple tool

Aiming at the implementation in Nanometer CMOS technologies, many efforts have been devoted to choose the $\Delta \Sigma$ topology suitable for the $\Delta \Sigma$ ADC design. A detailed analysis has been presented in [23], leading to optimized loop coefficients and performance for various FF $\Delta \Sigma$ modulators architectures. Optimized loop coefficients have been developed from behavioral simulations to stabilize high performance $\Delta \Sigma$ modulator. The loop coefficients represent the analog coefficients $\left(a_{i}\right)$, the feedforward coefficients $\left(c_{j}\right)$ and the digital coefficients $\left(e_{k}\right)$. The adopted prediction method is based on the following steps given by Fig. 2. In fact, we start with introducing some constants such as the signal bandwidth (BS), the $F_{\mathrm{S}}$, the supply voltage $\left(V_{\mathrm{dd}}\right)$, the analog and digital coefficients $\left(a_{i}\right.$ and $\left.e_{k}\right)$, the order $(L)$, the OverLoad $(O L)$, the quantizer bits number $(B)$ and the estimated value of the FF $\Delta \Sigma$ modulator power consumption $\left(P_{\text {mod }}\right)$, taking Tab. 1 and Tab. 2 into consideration. After that, the equations models related to FF $\Delta \Sigma$ modulator performance (equations from Eq. (1) to Eq. (10)) are used to present the trend of different singlebit FF $\Delta \Sigma$ modulators performance scaling from $350 \mathrm{~nm}$ to $22 \mathrm{~nm}$ process nodes. As an example, we are looking for designing the 2-2 cascaded FF $\Delta \Sigma$ modulator for DVB-H, WLAN and WIMAX standards. Thus, the loop coefficients introduced in the prediction method are taken from Tab. 3 [23].

The analog and the digital coefficients are respectively given by Eq. (23) and Eq. (24):

$$
\begin{aligned}
& a_{i}=\frac{C_{\mathrm{S}}}{C_{\mathrm{f}}} \text { where } 1 \leq i \leq 4, \\
& e_{2}=\frac{1}{e_{1} \cdot a_{1} \cdot a_{2}} .
\end{aligned}
$$

\begin{tabular}{|c|c|c|}
\hline $\begin{array}{l}\text { Loop coefficients } \\
\text { and performance }\end{array}$ & \multicolumn{2}{|c|}{$\begin{array}{c}\left(a_{1}, a_{2}, a_{3}, a_{4}, c_{1}, c_{2}, c_{3}, c_{4}, e_{1}, e_{2}\right) \\
(0.4,0.8,0.4,0.8,2,1,2,1,1,3.125)\end{array}$} \\
\hline OSR & $S N R_{\mathrm{p}}$ & $O L$ \\
\hline 16 & 65 & 0.95 \\
\hline 32 & 92 & 0.89 \\
\hline 64 & 121 & 0.88 \\
\hline 128 & 149 & 0.85 \\
\hline
\end{tabular}

Table 3 Optimized loop coefficients and performance for the single-bit cascaded

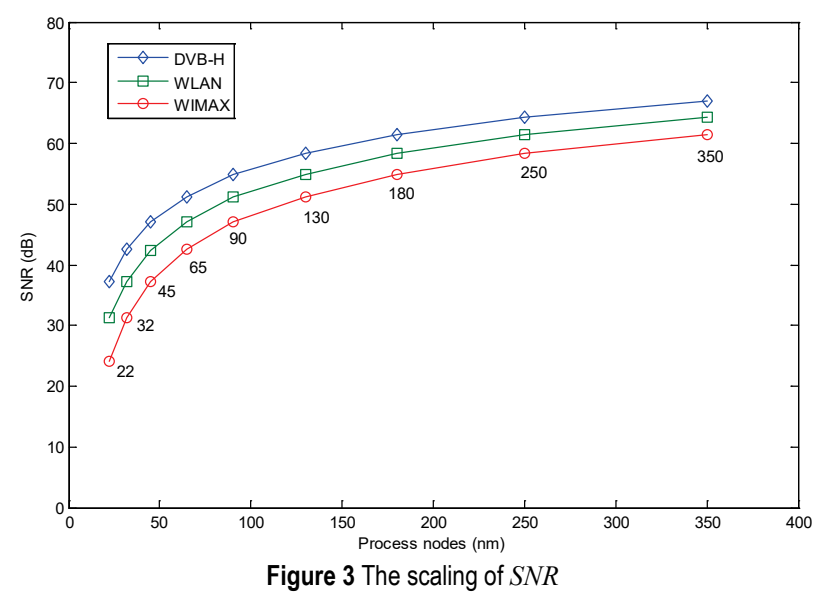

Figs. 3, 4, 5 and 6 show respectively the $S N R$, the ENOB, the power consumption and the FOM scaling of the 2-2 cascaded FF $\Delta \Sigma$ modulator for DVB-H, WLAN and WIMAX standards. Fig. 3 shows that the $S N R$ has been scaled from $67 \mathrm{~dB}$ to $37 \mathrm{~dB}$ when process node is scaling for DVB-H standard. From this figure, it has been remarked that the decrease of $S N R$ is due to the $F_{\mathrm{S}}$ reduction when scaling the device sizes. We notice a tradeoff between a higher bandwidth and a lower SNR. In Fig. 4 , due to the $S N R$ reduction, the value of the resolution decreases from 11bits. Fig. 6 shows that the FOM remains less than $0.26 \mathrm{pJ} /$ conversion when the channel length becomes shorter. Regarding the required human effort for the $\Delta \Sigma$ modulator performance prediction, the time spent is very large. This prompted us to propose a novel method that permits a fast $\Delta \Sigma$ modulator automatic design. 


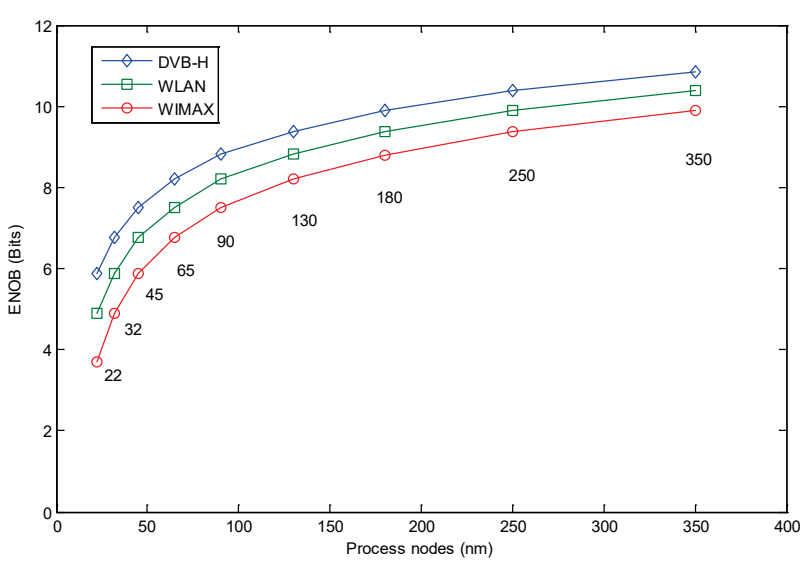

Figure 4 The scaling of $E N O B$

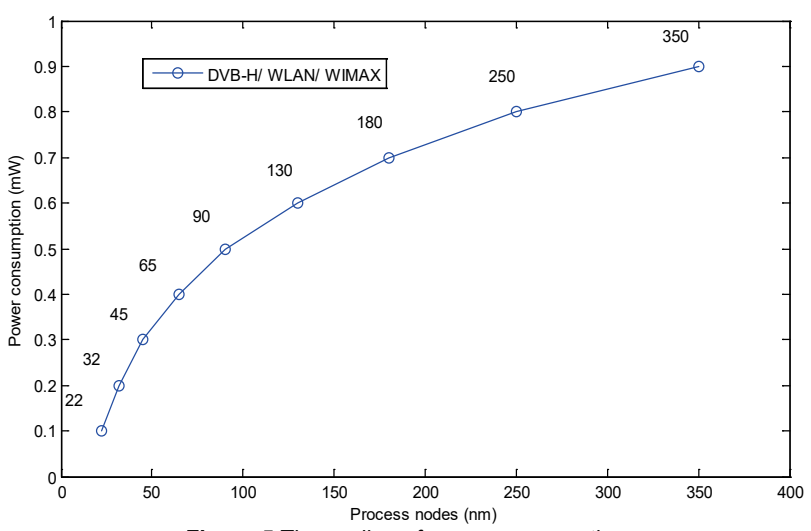

Figure 5 The scaling of power consumption

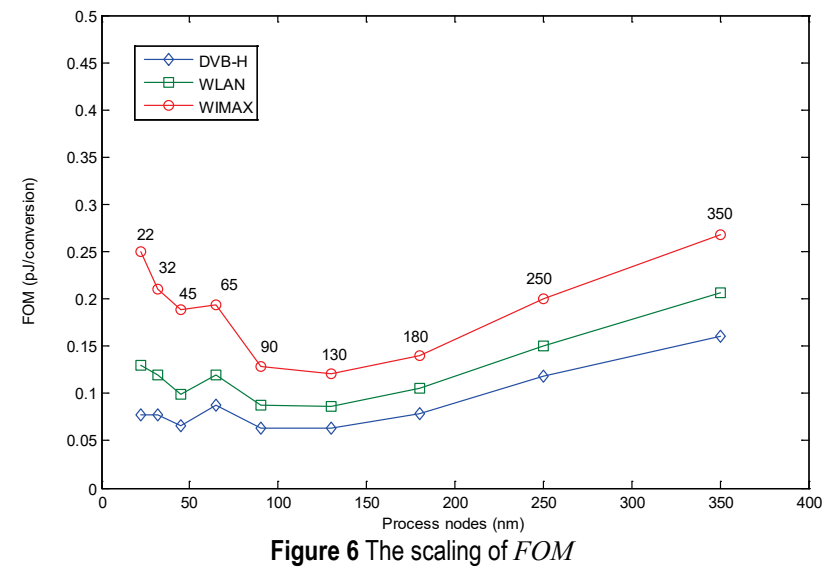

\section{AUTOMATED SYNTHESIS TOOL}

Our objective is to design different $\Delta \Sigma$ modulators topologies using future process without losing time. For this, we propose a new approach which is based on an algorithm to synthesize low power DT FF $\Delta \Sigma$ modulators.

\subsection{Proposed Synthesis Algorithm}

This algorithm is developed with "ActionScript 3" language while considering steps listed in Fig. 7. First, we start by setting the wireless standard and the $F_{\mathrm{S}}$ variations ranges listed in Tab. 1 and 2. Then, we choose power consumption less than a maximum default value of $1 \mathrm{~mW}$.

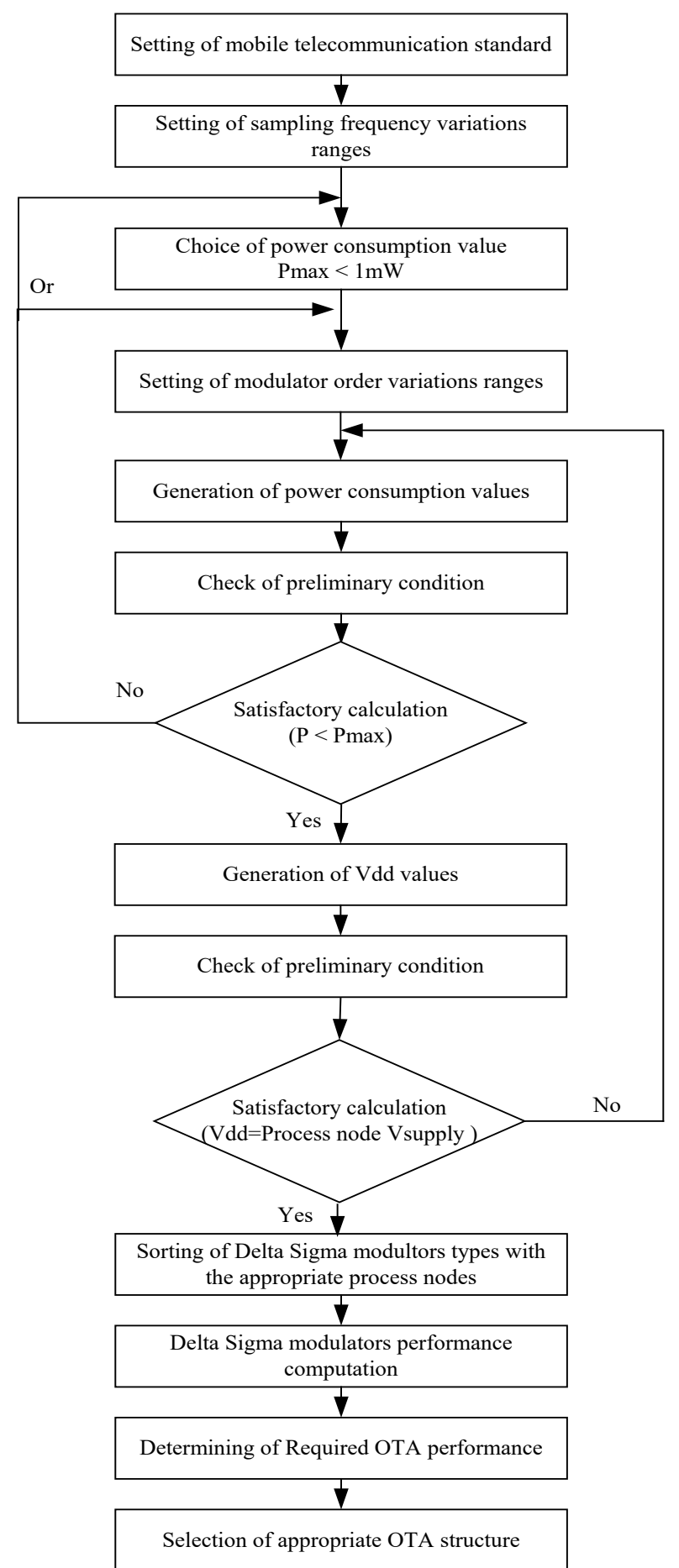

Figure 7 Proposed algorithm for $\mathrm{FF} \Delta \Sigma$ modulators automatic design

Next, we indicate the modulator order " $L$ " variations ranges. In this case, we choose to predict single loop (SL) $2^{\text {nd }}$ order modulators $(L=2), 2-1$ and 2-1-1 cascaded modulators $(L=3,4)$. By using the Eq. (8), all possible values of power consumption are generated followed by a verification of preliminary conditions. This condition consists of checking whether the calculated power consumption of the modulator is less than the maximum power value $P_{\max }$ which is initially selected. After that, the generation of possible power consumption values yields to possible generated supply voltages values followed by a verification of preliminary conditions. These conditions are imposed to ensure that the generated supply voltage is equal to the process node supply voltage given by Tab. 2 . 
If these conditions are fulfilled, the supply voltage is saved and it is a candidate for the following steps, otherwise we do another choice. For each power consumption and process node, a FF $\Delta \Sigma$ modulator type is then defined. The computation of its performance according to their developed equations models (Eq. (1) to Eq. (10)) yields to projected predictions. The characterization of OTA circuit depending on $\Delta \Sigma$ modulator performance is used to calculate the required performance for each designed modulator structure and to find the best adopted OTA architecture (Eq. (13)- Eq. (18)- Eq. (22)).

\subsection{FF $\Delta \Sigma$ Modulators Design Examples via Programming GUI}

Designing a good User Interface encourages an easy and natural interaction between a user and a system. A graphical application tool is a software program that graphically provides a user with a set of commands and icons with which he can graphically manipulate to generate or modify a design product in a work space area.

The proposed tool provides a wide design environment for designing different FF $\Delta \Sigma$ modulators. In fact, a GUI is programmed with "ActionScript 3" language where the user can easily include the data and display the results. In this subsection, we illustrate the design automation approach with some examples. Tab. 4 lists some required input parameters that the user should define at the beginning. In these examples, we are looking for designing FF $\Delta \Sigma$ modulators structures projected for UMTS, WLAN and WIMAX standards. From Fig. 8, it is seen that the user of the tool can select which standard to take into account during the prediction and set values for their lower and upper bounds.

Table 4 Input parameters to the design examples

\begin{tabular}{|c|c|c|c|}
\hline Standard & $B S(\mathrm{MHz})$ & $F_{\mathrm{S}}(\mathrm{MHz})$ & $P_{\text {mod }}(\mathrm{mW})$ \\
\hline UMTS & 2 & 100 & $<0.7$ \\
\hline WLAN & 10 & 200 & $<0.4$ \\
\hline WIMAX & 20 & 320 & $<0.3$ \\
\hline
\end{tabular}

After defining the design specification, the user waits for possible FF $\Delta \Sigma$ modulators architectures. In fact, the algorithm looks for candidate architectures with the given specifications. Fig. 9 displays various FF $\Delta \Sigma$ modulators topologies predicted for WIMAX standard. For each designed topology, the $\Delta \Sigma$ modulator performance is then computed. Among the availability of many structures, Tab. 5 presents predicted performance of the candidates architectures solutions for each given standard. Fig. 10 presents the corresponding required OTA performance for the provided 2-1 cascaded FF $\Delta \Sigma$ modulator given by Fig. 9. From Tab. 5, it can be concluded that the larger the value of modulator order, the higher the $S N R$ is. In fact, the highest $S N R$ value is achieved for 2-1-1 cascaded configuration. One of the important features of the design automation tool is the ability to find the best OTA structure for the provided FF $\Delta \Sigma$ modulators (Tab. 6). From Tab. 6, it is clear that for a given set of performance specifications, more than one topology is often feasible. In addition, the required type of OTA circuit can be provided for each calculated OTA performance.

Enter design specifications

\begin{tabular}{|c|c|c|c|c|}
\hline \multirow{2}{*}{$\begin{array}{l}\text { STANDARD } \\
\mathrm{BW}=20 \mathrm{MHz}\end{array}$} & ○ GSM & () UMTS & () DVB-H & () WIMAX \\
\hline & O Bluetooth & WCDMA & O WLAN & \\
\hline \multicolumn{2}{|c|}{ Sampling Frequency $(\mathrm{MHz})$} & \multicolumn{2}{|c|}{$F s=320$} & $200 \leq F s \leq 520$ \\
\hline \multicolumn{2}{|c|}{ Power consumption ( $\mathrm{mW}$ ) } & \multicolumn{2}{|c|}{$P<\quad 0.3$} & \\
\hline
\end{tabular}

Figure 8 GUI: specifications design

Due to the higher constraint on DC gain, the tool selects an OTA gain-boosted for 2-1-1 cascaded configuration for UMTS standard and a telescopic OTA for the rest.

Table 5 Designed single bit FF $\Delta \Sigma$ modulators structures projected for different standards

\begin{tabular}{|c|c|c|c|c|c|c|}
\hline \multirow{2}{*}{ Standard } & \multirow{2}{*}{ Configuration } & \multirow{2}{*}{ Process node (nm)/ Supply voltage (V) } & \multirow{2}{*}{$P_{\text {mod }}(\mathrm{mW})$} & \multicolumn{3}{|c|}{ Predicted performance } \\
\hline & & & & $S N R(\mathrm{~dB})$ & $E N O B$ (Bits) & $F O M(\mathrm{pJ} / \mathrm{conv})$ \\
\hline \multirow{2}{*}{ UMTS } & SL $2^{\text {nd }}$ order & $90 / 1.2$ & 0.52 & 57.86 & 9.32 & 0.203 \\
\hline & $2-1-1$ & $45 / 0.86$ & 0.25 & 92.54 & 15.08 & 0.018 \\
\hline \multirow{2}{*}{ WLAN } & $2-1$ & $65 / 1.2$ & 0.39 & 40.81 & 6.49 & 0.217 \\
\hline & $2-2$ & $32 / 0.76$ & 0.21 & 51.17 & 8.21 & 0.035 \\
\hline \multirow{2}{*}{ WIMAX } & $2-1$ & $45 / 0.86$ & 0.21 & 34.02 & 5.36 & 0.127 \\
\hline & $2-1-1$ & $22 / 0.68$ & 0.18 & 48 & 7.68 & 0.021 \\
\hline
\end{tabular}

Table 6 Required OTA performance for the provided FF $\Delta \Sigma$ modulators

\begin{tabular}{|c|c|c|c|c|c|c|}
\hline \multirow{2}{*}{ Standard } & \multirow{2}{*}{$\begin{array}{c}\text { Provided configuration } \\
\text { Process node }(\mathrm{nm}) / \text { Supply voltage }(\mathrm{V})\end{array}$} & \multicolumn{4}{|c|}{ Required OTA performance } & \multirow{2}{*}{ Selected OTA } \\
\hline & & $A_{\mathrm{dc}, \min }(\mathrm{dB})$ & $G B W_{\min }(\mathrm{GHz})$ & $S R_{\min }(\mathrm{V} / \mu \mathrm{s})$ & $P_{\text {OTA }}(\mathrm{mW})$ & \\
\hline \multirow{2}{*}{ UMTS } & $\begin{array}{c}\text { SL } 2^{\text {nd }} \text { order } \\
90 / 1.2\end{array}$ & 64.4 & 0.84 & 104.6 & 0.26 & Telescopic \\
\hline & $\begin{array}{c}\text { 2-1-1 Cascaded } \\
45 / 0.86\end{array}$ & 99.7 & 1.46 & 143.8 & 0.063 & Gain-boosted \\
\hline \multirow{2}{*}{ WLAN } & $\begin{array}{l}\text { 2-1 Cascaded } \\
65 / 1.2\end{array}$ & 48.6 & 1.35 & 256.4 & 0.13 & Telescopic \\
\hline & $\begin{array}{c}2-2 \text { Cascaded } \\
32 / 0.76 \\
\end{array}$ & 58.3 & 1.59 & 164.3 & 0.053 & Telescopic \\
\hline \multirow{2}{*}{ WIMAX } & $\begin{array}{l}\text { 2-1 Cascaded } \\
45 / 0.86\end{array}$ & 41.8 & 1.78 & 242.8 & 0.07 & Telescopic \\
\hline & $\begin{array}{l}2-1-1 \text { Cascaded } \\
22 / 0.68\end{array}$ & 55.18 & 2.38 & 185.3 & 0.045 & Telescopic \\
\hline
\end{tabular}




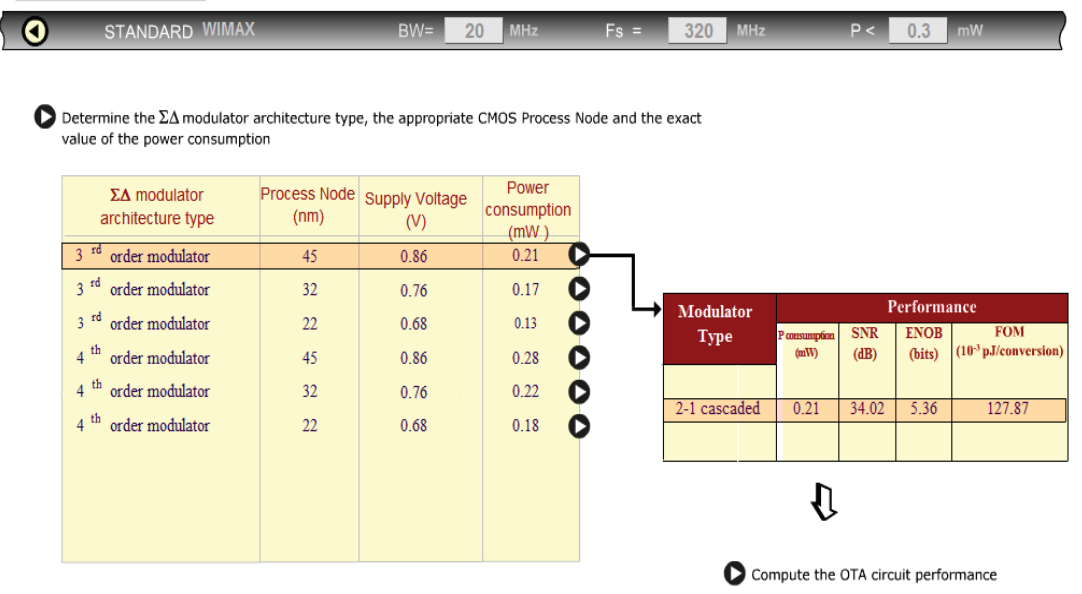

Figure 9 GUI: predicted performance of different modulators architectures for WIMAX standard

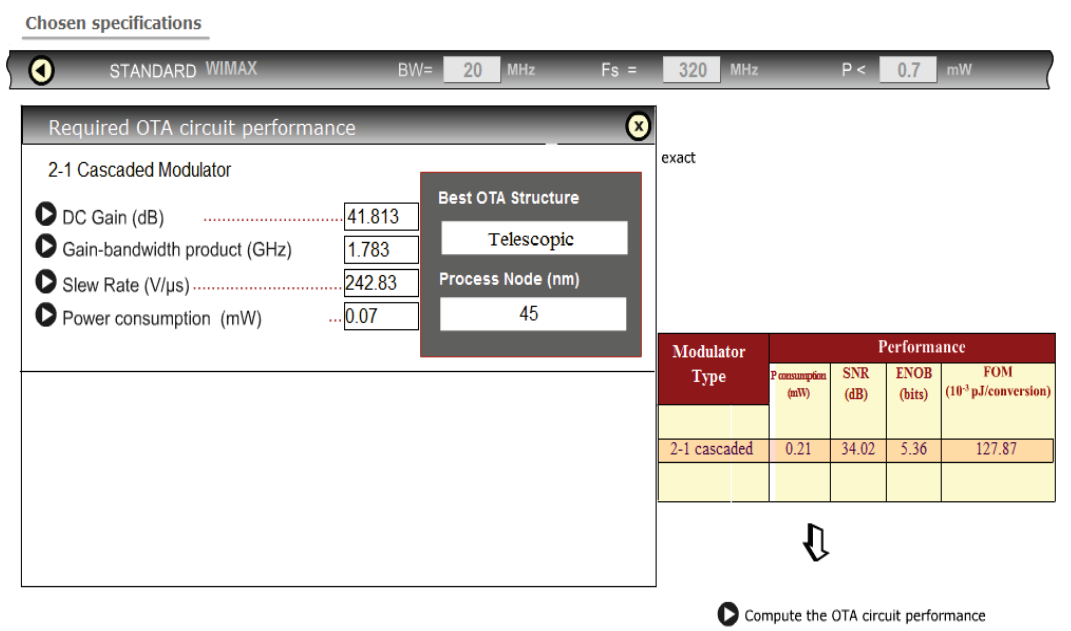

Figure $10 \mathrm{GUI}$ : predicted performance of required OTA for WIMAX standard

Table 6 Required OTA performance for the provided FF $\Delta \Sigma$ modulators

\begin{tabular}{|c|c|c|c|c|c|c|}
\hline \multirow{2}{*}{ Standard } & \multirow{2}{*}{$\begin{array}{c}\text { Provided configuration } \\
\text { Process node }(\mathrm{nm}) / \text { Supply voltage }(\mathrm{V})\end{array}$} & \multicolumn{4}{|c|}{ Required OTA performance } & \multirow{2}{*}{ Selected OTA } \\
\hline & & $A_{\mathrm{dc}, \min }(\mathrm{dB})$ & $G B W_{\min }(\mathrm{GHz})$ & $S R_{\min }(\mathrm{V} / \mu \mathrm{s})$ & $P_{\mathrm{OTA}}(\mathrm{mW})$ & \\
\hline \multirow{2}{*}{ UMTS } & $\begin{array}{c}\text { SL } 2^{\text {nd }} \text { order } \\
90 / 1.2\end{array}$ & 64.4 & 0.84 & 104.6 & 0.26 & Telescopic \\
\hline & $\begin{array}{l}\text { 2-1-1 Cascaded } \\
45 / 0.86\end{array}$ & 99.7 & 1.46 & 143.8 & 0.063 & Gain-boosted \\
\hline \multirow{2}{*}{ WLAN } & $\begin{array}{l}\text { 2-1 Cascaded } \\
65 / 1.2\end{array}$ & 48.6 & 1.35 & 256.4 & 0.13 & Telescopic \\
\hline & $\begin{array}{l}2-2 \text { Cascaded } \\
32 / 0.76\end{array}$ & 58.3 & 1.59 & 164.3 & 0.053 & Telescopic \\
\hline \multirow{2}{*}{ WIMAX } & $\begin{array}{l}2-1 \text { Cascaded } \\
45 / 0.86\end{array}$ & 41.8 & 1.78 & 242.8 & 0.07 & Telescopic \\
\hline & $\begin{array}{l}\text { 2-1-1 Cascaded } \\
22 / 0.68\end{array}$ & 55.18 & 2.38 & 185.3 & 0.045 & Telescopic \\
\hline
\end{tabular}

\section{CONCLUSION AND FUTURE EXTENSIONS}

This paper proposed a novel helpful approach for the synthesis of low power FF $\Delta \Sigma$ modulators with process scaling for various mobile telecommunications standards. The procedure of NanoCMOS $\Delta \Sigma$ modulators performance prediction and the use of the MAPLE tool have been presented. Automation of predicting $\Delta \Sigma$ modulators performance is a very challenging and time-consuming task. Thus, a novel automated synthesis tool, which includes FF $\Delta \Sigma$ modulators performance synthesis and OTA performance modeling, has been proposed. A new algorithm has been suggested to provide the prediction of single-bit FF $\Delta \Sigma$ modulators performance for wireless standards. The presented approach proves that the design effort is reduced with the assistance of an automated tool. Some design examples have been presented through a programmed Graphical User Interface. This GUI was designed allowing convenient and fast access to all functionality. These examples showed that the proposed tool may be used to generate different FF $\Delta \Sigma$ modulators structures types for desired specifications. It has shown that higher SNR can be reached for high order low power modulator topology. This tool reveals the link between both the design and the technological parameters and structures performance to provide an insight to the $\Delta \Sigma$ modulator design problem and lightens up the design environment for the designer. The outcome of the synthesis tool is not only the projected $\Delta \Sigma$ modulator performance, but also the required building block specifications. The 
proposed tool is beneficial in predicting the FF $\Delta \Sigma$ modulators performance and the required OTA specifications. It can further shorten the design time for inexperienced designers. The automated synthesis tool can be used to predict the performance of a wide variety of analog circuits and topologies.

In the future, to raise the efficiency of design automation systems, developed analytical models incorporating circuit non-idealities will be introduced further in the proposed synthesis tool. In addition, an extension of this research might be the prediction of transistor sizes of analog circuits for upcoming process using a Nano-CMOS device-level tool to increase its usability

\section{REFERENCES}

[1] Lee, K., Miller, M. R., \& Temes, G. C. (2009). An $8.1 \mathrm{~mW}$, $82 \mathrm{~dB}$ delta-sigma ADC with $1.9 \mathrm{MHz}$ BW and $98 \mathrm{~dB}$ THD. IEEE Journal of Solid-State Circuits, 44(8), 2202-2211. https://doi.org/10.1109/JSSC.2009.2022298

[2] Nam, K. Y., Lee, S.-M., Su, D. K., \& Wooley, B. A. (2005). A low-voltage low-power sigma-delta modulators for broadband analog-to-digital conversion. IEEE Journal of Solid State Circuits, 40(9), 1855-1864. https://doi.org/10.1109/JSSC.2005.852161

[3] Anand, A., Koirala, N., Pokharel, R. K., Kanaya, H., \& Yoshida, K. (2013). Systematic design methodology of a wideband multibit continuous time delta-sigma modulator. International Journal of Microwave Science and Technology, 1-5. https://doi.org/10.1155/2013/275289

[4] De la Rosa, J. M., Schreier, R., Pun, K- P., \& Pavan, S. (2015). Next generation delta sigma converters: trends and perspectives. IEEE Journal on Emerging and Selected Topics in Circuits and Systems, 5(4), 484-499. https://doi.org/10.1109/JETCAS.2015.2502164

[5] Yagyu, M. \& Nishihara, A. (2004). Fast and efficient algorithm to design noise-shaping FIR filters for high-order overload-free stable sigma-delta modulators. Proceedings of the IEEE Int. Symposium on Circuits and Systems / Canada, 469-472.

[6] Wei, Y., Tang, H., \& Doboli, A. (2007). Systematic Methodology for Designing Reconfigurable $\Delta \Sigma$ Modulator Topologies for Multimode Communication Systems. IEEE Transactions on Computer-Aided Design of Integrated Circuits and Systems, 26(3), 480-496. https://doi.org/10.1109/TCAD.2006.885734

[7] Medeiro, F., de la Rosa, J. M., Pérez-Verdú, B., \& Rodríguez-Vázquez, A. (2012). Analog Circuit Design: MOST RF Circuits, Sigma-Delta Converters and Translinear Circuits. Springer Science \& Business Media, 231-253.

[8] Tang, H. \& Doboli, A. (2006). High-level synthesis of $\Sigma \Delta$ modulator topologies optimized for complexity, sensitivity, and power consumption. IEEE Transactions on ComputerAided Design of Integrated Circuits and Systems, 25(3), 597607. https://doi.org/10.1109/TCAD.2005.854633

[9] Rusu, A. \& Ismail, M. (2005). Low-distortion band pass $\Delta \Sigma$ modulator for wireless radio receivers. Electronics letters. 41(19), 1044-1046. https://doi.org/10.1049/el:20052167

[10] Del Río, R. (2006). CMOS cascade sigma-delta modulators for sensors and telecom: error analysis and practical design. Springer.

[11] Pavan, S., Schreier, R., \& Temes, G. C. (2017). Understanding delta-sigma data converters. John Wiley \& Sons.

[12] Feldman, A. R. (1997). High-speed, low-power sigma-delta modulators for RF baseband channel applications. Thesis, University of California, Berkeley.
[13] Porrazzo, S., Morgado, A., Segundo Bello, S., van Hoof, D., Yazicioglu, F., van Roermund, C. R., A. H. M., \& Cantatore, E. (2015). A design methodology for power-efficient reconfigurable SC $\Sigma \Delta$ modulators. International Journal of Circuit Theory and Applications, 43(8), 1024-1041. https://doi.org/10.1002/cta.1992

[14] Yang, Y. (2008). System oriented delta sigma analog-todigital modulator design for ultra-high precision data acquisition applications. University of Texas.

[15] Aminzadeh, H., Lotfi, R., \& Mafinezhad, K. (2009). Design of low-power single-stage operational amplifiers based on an optimized settling model. Analog Integrated Circuits and Signal Processing, 58(2), 153-160. https://doi.org/10.1007/s10470-008-9226-1

[16] Waltari, M. (2002). Circuit techniques for low-voltage and high-speed A/D converters. Thesis, Helsinki University of Technology.

[17] Bult, K. \& Geelen, G. J. G. M. (1990). A Fast-settling CMOS OP AMP for SC circuits with 90-dB DC gain. IEEE Journal of Solid-State Circuits, 25(6), 1379-1384. https://doi.org/10.1109/4.62165

[18] Li, X. \& Ismail, M. (2002). Multi-standard CMOS wireless receivers: analysis and design. Springer Science \& Business Media.

[19] Silva, A., Guilherme, J., \& Horta, N. (2009). Reconfigurable multi-mode sigma-delta modulator for $4 \mathrm{G}$ mobile terminals. VLSI Journal, 42(1), 34-46. https://doi.org/10.1016/j.vlsi.2008.07.004

[20] Jose, R., Mathew, J., \& Mythili, P. (2009). A Multi-mode sigma-delta ADC for GSM/WCDMA/WLAN applications. Journal of Signal Processing Systems, 62(2), 117-130. https://doi.org/10.1007/s11265-008-0326-z

[21] Ke, Y., Craninckx, J., \& Gielen, G. (2008). A design approach for power-optimized fully reconfigurable $\Sigma \Delta \mathrm{A} / \mathrm{D}$ converter for $4 \mathrm{G}$ radios. IEEE Transactions on Circuits and Systems: Express Briefs, 55(3), 229-233.

[22] Daoud, H., Ben Salem, S., Zouari, S., \& Loulou, M. (2012). Use of robust method for NanoCMOS process prediction application to basic block analog circuit design. Journal of Circuits Systems and Computers, 21(7), 22. https://doi.org/10.1142/S0218126612500612

[23] Yao, L., Steyaert, M., \& Sansen, W. (2009). Low-power lowvoltage sigma-delta modulators in nanometer CMOS. Springer.

\section{Contact information}

Houda DAOUD, Assistant professor

(Corresponding author)

National School of Electronics and Telecommunications of Sfax National Engineering School of Sfax, B.P.W., Sfax, Tunisia houda.daoud@enetcom.usf.tn

Samir Ben SALEM, Assistant professor

National School of Electronics and Telecommunications of Sfax National Engineering School of Sfax, B.P.W., Sfax, Tunisia samir.bensalem@enetcom.usf.tn

Sawssan LAHIANI, PhD student

National School of Electronics and Telecommunications of Sfax National Engineering School of Sfax, B.P.W., Sfax, Tunisia sawssenlahiani@yahoo.fr

Mourad LOULOU, Professor

National Engineering School of Sfax

National Engineering School of Sfax, B.P.W., Sfax, Tunisia mourad.loulou@ieee.org 